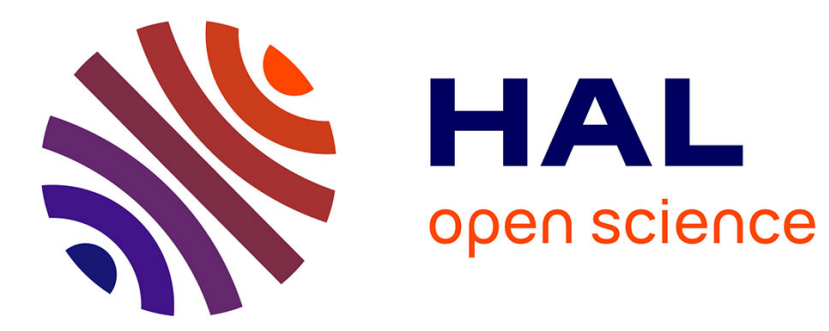

\title{
Uzawa Block Relaxation Methods for Color Image Restoration
}

Cédric Loosli, Stéphanie Jehan-Besson, Jonas Koko

\section{To cite this version:}

Cédric Loosli, Stéphanie Jehan-Besson, Jonas Koko. Uzawa Block Relaxation Methods for Color Image Restoration. CPCV 2012: ECCV workshop: Color and Photometry in Computer Vision, Oct 2012, Florence, Italy. pp.492-503, 10.1007/978-3-642-33868-7_49 . hal-00862158

\section{HAL Id: hal-00862158 https://hal.science/hal-00862158}

Submitted on 16 Sep 2013

HAL is a multi-disciplinary open access archive for the deposit and dissemination of scientific research documents, whether they are published or not. The documents may come from teaching and research institutions in France or abroad, or from public or private research centers.
L'archive ouverte pluridisciplinaire HAL, est destinée au dépôt et à la diffusion de documents scientifiques de niveau recherche, publiés ou non, émanant des établissements d'enseignement et de recherche français ou étrangers, des laboratoires publics ou privés. 


\title{
Uzawa block relaxation methods for color image restoration
}

\author{
Cédric Loosli ${ }^{1}$, Stéphanie Jehan-Besson ${ }^{2}$, and Jonas Koko ${ }^{1}$ \\ ${ }^{1}$ LIMOS, Université Blaise Pascal - CNRS UMR 6158, Complexe des Cézeaux, 63173 AUBIERE \\ 2 GREYC Laboratory, CNRS UMR 6072, Bd du Maréchal Juin, 14050 Caen, FRANCE \\ cloosli@isima.fr, jehan@ensicaen.fr, koko@sp.isima.fr
}

\begin{abstract}
In this paper we propose to investigate the use of a vectorial total variation model with spatially varying regularization and data terms for color image denoising and restoration. We pay attention to two main minimization problems: the minimization of a weighted vectorial total variation term $T V_{g}$, which acts as a regularization term, using the $L^{2}$ norm as data term or the minimization of the vectorial total variation with a spatially varying $L_{g}^{1}$ norm. The optimization process takes benefit of convex optimization tools by introducing an augmented Lagrangian formulation. This formulation leads us to simple and efficient algorithms based on Uzawa block relaxation schemes that are also robust towards the choice of the penalty parameter. In this paper, We propose to study more particularly the impact of spatially varying terms (total variation term or data terms) for color image restoration. A new weighted total variation term is proposed for old parchments restoration and we also compare the use of a weighted total variation term with a spatially varying data term for impulse noise removal in color images.
\end{abstract}

\section{Introduction}

In this paper, we are interested in addressing the vectorial (color) image restoration problem through the minimization of a unique criterion that takes benefit of a vectorial total variation term acting as a regularization term. Such a criterion proves to be interesting notably to cope with the regularization of correlated vectorial image features and may avoid to find an appropriate color space change when dealing with color image restoration. Let first remind that the importance of total variation for image restoration has been largely proved since the seminal ROF model introduced by Rudin, Osher and Fatemi [1]. In this work, the authors propose to recover the restored image $u$ from a noisy image $f$ by minimizing the following criterion :

$$
E(u)=\int_{\Omega}|\nabla u(x)| \mathrm{d} x+\lambda \int_{\Omega}|f(x)-u(x)|^{2} \mathrm{~d} x
$$

where $\Omega$ is the image domain, $u: \Omega \rightarrow R$ is the unknown restored image, $f$ is the observed image and $\lambda$ a positive scale parameter. The model (1.1) is also called $T V+L^{2}$ model. Such a framework has been intensively investigated for denoising [1,2]. It has also been extended by changing the $L^{2}$ norm by a $L^{1}$ norm $\left(T V+L^{1}\right.$ model) for salt and pepper noise removal (see for example [3]), texture extraction or decomposition (e.g. [4]) or shape denoising (e.g. [5]). The minimization issues of such problems are not trivial due to the non differentiability of the total variation regularization term and also of the $L^{1}$ norm and have been addressed by many authors. For example, standard calculus of variations and Euler-Lagrange equations can be used to compute the PDE that will drive the functional $u$ towards a minimum of $E$. This method requires a smooth approximation of the $L^{1}$ norm and a small time step must be chosen so as to ensure the convergence. This often leads to a large number of iterations as mentioned in [5]. 
In [6], a MRF (Markov Random Field) model is proposed which uses the anisotropic separable approximation (i.e. $|\nabla u|=\left|D_{x} u\right|+\left|D_{y} u\right|$ where $D_{x}$ and $D_{y}$ are the horizontal and vertical discrete derivative operators). This approximation is also used in [7] where the authors proposed an efficient graph-cut method. In all these approaches, an approximation or a smoothing of the $L^{1}$ norm is required. In [5,8], based on the works of $[2,9,10,4]$, a fast minimization algorithm based on a dual formulation is proposed for the minimization of $T V+L^{1}$. Thanks to such approaches, they do not need any approximation or smoothing of the $L^{1}$ norm, they rather take benefit of a convex regularization of the criterion which was first proposed by [4]. More recently, a lot of very efficient numerical methods using convex analysis tools have been proposed. Among them, we can cite the primal-dual method proposed in [2], the split Bregman method [11] or the unified framework proposed by Pock and Chambolle [12]. Such algorithms have become popular due to their low computational cost. In this paper we also take benefit of convex optimization tools by using a simple and efficient algorithm based on Uzawa block relaxation schemes and an augmented Lagrangian formulation $[13,14]$. This scheme is efficient and have the nice property to be robust towards the choice of the penalty parameter. Moreover, it is here computed and applied in the special case of vectorial (color) image restoration.

When dealing with multi-components images (such as color images), the image $f$ becomes a vector with $n$ components (e.g. $\mathbf{f}=\left(f_{1}, f_{2}, f_{3}\right)^{T}$ and $n=3$ for color images) which leads to revise the above criterion and to propose a well-adapted definition of the vectorial total variation term. In a first work, Blomgren and Chan [15] propose to restore vector-valued images using a vectorial adaptation of the total variation term. Later, some other color total variation models have been proposed by different authors (see for example $[16,17]$ ) for color image restoration. In $[16,17]$ the vectorial total variation term to minimize becomes an integral over a function of the larger and smaller eigenvalues of the structure tensor proposed by di Zenso [18] (see also [19] for a recent review on anisotropic color diffusion PDEs). When the function is chosen to be the identity, their scheme leads to the minimization of the following regularization term $\int_{\Omega}(\|\nabla \mathbf{u}\|) \mathrm{d} x$ where $\|\nabla \mathbf{u}(x)\|=\left(\sum_{i=1}^{n}\left|\nabla u_{i}(x)\right|^{2}\right)^{1 / 2}$ is the Frobenius norm of the derivative $\nabla \mathbf{u}$. This vectorial total variation regularization term has then proven to be convenient when dealing with minimization using dual approaches [20-23] and leads to good results for color restoration due to its link to the eigenvalues of the structure tensor. However, this regularization term fails in preserving sharp details like edges and texture regions.

In order to circumvent such problems, recent works (see for example [24] for color restoration) propose some spatially varying terms in the functional to minimize. The idea is to weight the data term differently according to the localisation in the image by minimizing a spatially adaptive functional of the form:

$$
E(\mathbf{u})=\int_{\Omega}\|\nabla \mathbf{u}(x)\| \mathrm{d} x+\frac{1}{\tau} \int_{\Omega} \lambda(x)|\mathbf{f}(x)-\mathbf{u}(x)|^{\tau}
$$

with $\tau \in[1,2]$. Here, the function $\lambda(x)$ is similar to the constant $\lambda$ but is calculated on each point of the image. For example, a recent work [24] establishes a general framework for color image restoration dealing with both the $L^{2}$ and $L^{1}$ cases. It is solved by Fenchel-duality and semi-smooth Newton techniques. Some other works rather propose to deal with a weighted total variation term as for example in [25] for color image restoration or $[5,26,8]$ for grey level image restoration.

In this work, we then propose to study spatially varying regularization and data terms for color image restoration. We first solve the minimization of the following vectorial $T V_{g}+L^{\tau}$ model:

$$
E(\mathbf{u})=\int_{\Omega} g(x)\|\nabla \mathbf{u}(x)\| \mathrm{d} x+\lambda \int_{\Omega}|\mathbf{f}(x)-\mathbf{u}(x)|^{\tau} \mathrm{d} x
$$


where $\Omega$ is the image domain, $\mathbf{u}: \Omega \rightarrow \mathbb{R}^{3}$ is the restored image, $\mathbf{f}$ is the observed image and $g: \Omega \rightarrow] 0, A](A>0)$ is a function chosen according to the application or the noise model. In this paper, for space reasons, we propose the minimization scheme only for $\tau=2$ but the minimization for the $L^{1}$ norm can be performed using the same mathematical framework as proposed in [8]. The constant $\lambda>0$ is a positive scale parameter. Such a spatially varying regularization term appears to be interesting both for Gaussian [5] using the $L^{2}$ norm and salt and pepper denoising $[27,8]$ by using a well adapted function $g$ and the $L^{1}$ norm. Here, we propose an adapted function $g$ for the restoration of old parchments. One may ask whether it is more interesting to use a spatially varying data term or a spatially varying regularization term. Indeed, it depends on the application. The spatially varying regularization term seems better adapted for a structural filtering (edges preservation or removing components according to their structure) while the spatially data term seems better adapted for a syntactic filtering and can better preserve the intensity of some pixels. In this paper, we then provide an example which attests the real interest of the $T V_{g}$ term for old parchments restoration but we also propose an example of impulse noise removal where a well designed spatially varying data term may be more interesting. To this end, we then propose to solve the minimization of the following criterion:

$$
E_{2}(\boldsymbol{u})=\lambda \int_{\Omega}\left[\left|\nabla u_{1}\right|^{2}+\left|\nabla u_{2}\right|^{2}+\left|\nabla u_{3}\right|^{2}\right]^{1 / 2} \mathrm{~d} x+\sum_{i=1}^{3} \int_{\Omega} g_{i}(x)\left|u_{i}(x)-f_{i}(x)\right| \mathrm{d} x,
$$

where $\mathbf{g}=\left[g_{1}, g_{2}, g_{3}\right]^{T}$ is now a vectorial function with different components according to the color channels. Experimental results on simulated noisy images (salt and pepper on each color channel) provide a first example where this spatially varying data term may be advantageously used.

The minimization problem and notations are given in section 2 while the Augmented Lagrangian formulation is detailed in section 3 for $T V_{g}+L^{2}$ and section 4 for $T V+L_{g}^{1}$. Experimental results are given in section 5 .

\section{Problem statement}

\subsection{Notations and minimization problems}

Let $\Omega$ be a three-dimensional bounded open domain of $\mathbb{R}^{d}, d=2,3$. We consider a vector-valued function $\boldsymbol{u}(x)=\left(u_{1}(x), u_{2}(x), u_{3}(x)\right) \in \mathbb{R}^{3}$ defined on $\Omega$. For a color image defined on $\Omega$, the components $u_{i}$ stand for the three values of each color channel (for example the RGB color space). To simplify, vector valued functions are denoted by bold-face letters (e.g. $\left.\boldsymbol{u}=\left(u_{1}, u_{2}, u_{3}\right)\right)$. The Euclidean scalar product is $\boldsymbol{u} \cdot \boldsymbol{v}=\sum_{i=1}^{d} u_{i} v_{i}$, for $\boldsymbol{u}$ and $\boldsymbol{v}$ in $\mathbb{R}^{d}$. Moreover, for $\boldsymbol{u} \in \boldsymbol{R}^{d}$, we use the notations: $|\boldsymbol{u}|_{2}=(\boldsymbol{u} \cdot \boldsymbol{u})^{1 / 2}, \quad|\boldsymbol{u}|_{1}=\sum_{i=1}^{d}\left|u_{i}\right|, \quad|\boldsymbol{u}|_{\infty}=\max _{i=1, \ldots, d}\left|u_{i}\right|$ for the Euclidean norm, the 1-norm and the infinity norm, respectively.

Let $\boldsymbol{f}=\left(f_{1}, f_{2}, f_{3}\right)$ be an observed (blurry or noisy) color image. We propose to address the two following minimization problems where $\boldsymbol{u}=\left(u_{1}, u_{2}, u_{3}\right)$ is the unknown image to restore and $\boldsymbol{V}$ is a suitable functions space (or a finite dimensional space):

- The $T V_{g}+L^{2}$ which is defined as follows :

$$
\min _{\boldsymbol{u} \in \boldsymbol{V}} E_{1}(\boldsymbol{u})=\int_{\Omega} g(x)\left[\left|\nabla u_{1}\right|^{2}+\left|\nabla u_{2}\right|^{2}+\left|\nabla u_{3}\right|^{2}\right]^{1 / 2} \mathrm{~d} x+\lambda \int_{\Omega}|\boldsymbol{u}(x)-\boldsymbol{f}(x)|_{2}^{2} \mathrm{~d} x,
$$

where $g$ is a scalar function. 
- The $T V+L_{g}^{1}$ model which is defined as follows :

$$
\min _{\boldsymbol{u} \in \boldsymbol{V}} E_{2}(\boldsymbol{u})=\lambda \int_{\Omega}\left[\left|\nabla u_{1}\right|^{2}+\left|\nabla u_{2}\right|^{2}+\left|\nabla u_{3}\right|^{2}\right]^{1 / 2} \mathrm{~d} x+\sum_{i=1}^{3} \int_{\Omega} g_{i}(x)\left|u_{i}(x)-f_{i}(x)\right| \mathrm{d} x,
$$

where $\mathbf{g}=\left[g_{1}, g_{2}, g_{3}\right]^{T}$ is a vectorial function.

In the case $T V_{g}+L^{2}$, we precise the first term. Let $g$ be a continuous, positive valued and bounded function defined on $\Omega$. Let us introduce the weighted total variation regularization term, denoted by $T V_{g}$, derived from [5]

$$
J(\boldsymbol{u})=\int_{\Omega} g(x)\left[\left|\nabla u_{1}\right|^{2}+\left|\nabla u_{2}\right|^{2}+\left|\nabla u_{3}\right|^{2}\right]^{1 / 2} \mathrm{~d} x=\sup _{\boldsymbol{\phi} \in \Phi_{g}}(\boldsymbol{u}, \nabla \cdot \phi)_{L^{2}\left(\Omega ; \mathbb{R}^{3}\right)}
$$

where $\Phi_{g}=\left\{\phi \in \mathcal{C}^{1}\left(\Omega, \mathbb{R}^{3}\right):|\phi(x)| \leq g\right.$, for all $\left.x \in \Omega\right\}$.

\section{Augmented Lagrangian methods for the $T V_{g}+L^{2}$ model}

In this section we present Uzawa (dual) methods for solving (2.1). To this end, we need to transform the convex minimization problem (2.1) into a suitable saddle-point problem by introducing an auxiliary unknown as for the scalar case [8]. For space reasons, only few elements are given.

\subsection{Augmented Lagrangian formulation}

Let us introduce the auxiliary unknown $\boldsymbol{p}=\boldsymbol{f}-\boldsymbol{u}$ and rewrite the functional $E_{1}$ as

$$
E_{1}(\boldsymbol{u}, \boldsymbol{p})=J(\boldsymbol{u})+\lambda \int_{\Omega}|p(x)|_{2}^{2} \mathrm{~d} x .
$$

The minimization problem (3.1) becomes

$$
\min _{(\boldsymbol{u}, \boldsymbol{p}) \in K} E_{1}(\boldsymbol{u}, \boldsymbol{p}),
$$

where the constraint set $K$ is defined by $K=\{(\boldsymbol{u}, \boldsymbol{p}) \in X \times X \mid \boldsymbol{u}+\boldsymbol{p}-\boldsymbol{f}=0$ in $\Omega\}$.

It is obvious that problems (3.1) and (3.2) are equivalent. To the constrained minimization problem (3.2) we associate the Lagrangian functional $\mathscr{L}$ defined on $X \times X \times X$ by

$$
\mathscr{L}(\boldsymbol{u}, \boldsymbol{p} ; \boldsymbol{s})=E_{1}(\boldsymbol{u}, \boldsymbol{p})+(\boldsymbol{s}, \boldsymbol{u}+\boldsymbol{p}-\boldsymbol{f})_{X} .
$$

In (3.3), $s$ is the Lagrange multiplier associated with the constraint in $K$. Since $E_{1}$ is convex and continuous, a saddle point $\left(\boldsymbol{u}^{*}, \boldsymbol{p}^{*} ; \boldsymbol{s}^{*}\right)$ of $\mathscr{L}$ exists and verifies $\mathscr{L}\left(\boldsymbol{u}^{*}, \boldsymbol{p}^{*} ; \boldsymbol{s}\right) \leq \mathscr{L}\left(\boldsymbol{u}^{*}, \boldsymbol{p}^{*} ; \boldsymbol{s}^{*}\right) \leq$ $\mathscr{L}\left(\boldsymbol{u}, \boldsymbol{p} ; \boldsymbol{s}^{*}\right), \quad \forall(\boldsymbol{u}, \boldsymbol{p}, \boldsymbol{s}) \in X \times X \times X$.

We now introduce the augmented Lagrangian defined, for $r>0$, by

$$
\mathscr{L}_{r}(\boldsymbol{u}, \boldsymbol{p} ; \boldsymbol{s})=\mathscr{L}(\boldsymbol{u}, \boldsymbol{p} ; \boldsymbol{s})+\frac{r}{2}\|\boldsymbol{u}+\boldsymbol{p}-\boldsymbol{f}\|_{L^{2}}^{2}
$$

where $r$ is the penalty parameter. It can be proved (easily) that a saddle point of $\mathscr{L}_{r}$ is a saddle point of $\mathscr{L}$ and conversely. This is due to the fact that the quadratic term in $\mathscr{L}_{r}$ vanishes when the constraint $\boldsymbol{u}+\boldsymbol{p}-\boldsymbol{f}=0$ is satisfied. Some efficient numerical schemes can be used to solve this problem like notably the Uzawa Block Relaxation method detailed thereafter. One important feature is that this algorithm is well conditioned against the choice of the penalty parameter $r$. 


\subsection{Uzawa block relaxation methods}

We apply Uzawa block relaxation methods to the augmented Lagrangian functional (3.4). We can give a symmetric role to the unknowns $\boldsymbol{u}$ and $\boldsymbol{p}$ by updating the multiplier $\boldsymbol{s}$ between Step 1 and Step 3. We obtain the following Uzawa block relaxation algorithm.

\section{Algorithm UBR}

Initialization. $\boldsymbol{p}^{-1}, \boldsymbol{s}^{0}$ and $r>0$ given.

Iteration $k \geq 0$. Compute successively $\boldsymbol{u}^{k}, \boldsymbol{p}^{k}$ and $\boldsymbol{s}^{k}$ as follows.

Step 1. Subproblem 1 : Find $\boldsymbol{u}^{k} \in X$ s.t. $\mathscr{L}_{r}\left(\boldsymbol{u}^{k}, \boldsymbol{p}^{k-1} ; \boldsymbol{s}^{k}\right) \leq \mathscr{L}_{r}\left(\boldsymbol{v}, \boldsymbol{p}^{k-1} ; \boldsymbol{s}^{k}\right), \quad \forall \boldsymbol{v} \in X$.

Step 2. Update the Lagrange multiplier $\boldsymbol{s}^{k+1 / 2}=\boldsymbol{s}^{k}+\frac{r}{2}\left(\boldsymbol{u}^{k}+\boldsymbol{p}^{k-1}-\boldsymbol{f}\right)$

Step 3. Subproblem 2 : Find $\boldsymbol{p}^{k} \in X$ s.t. $\mathscr{L}_{r}\left(\boldsymbol{u}^{k}, \boldsymbol{p}^{k} ; \boldsymbol{s}^{k}\right) \leq \mathscr{L}_{r}\left(\boldsymbol{u}^{k}, \boldsymbol{q} ; \boldsymbol{s}^{k}\right), \quad \forall \boldsymbol{q} \in X$.

Step 4. Update the Lagrange multiplier $\boldsymbol{s}^{k+1}=\boldsymbol{s}^{k+1 / 2}+\frac{r}{2}\left(\boldsymbol{u}^{k}+\boldsymbol{p}^{k}-\boldsymbol{f}\right)$

\subsection{Solution of subproblem 1}

The functional $\boldsymbol{u} \mapsto \mathscr{L}_{r}\left(\boldsymbol{u}, \boldsymbol{p}^{k-1} ; \boldsymbol{s}^{k}\right)$ can be rewritten as

$$
\Phi_{1}(\boldsymbol{u}):=\frac{r}{2}\|\boldsymbol{u}\|_{L^{2}}^{2}+J(\boldsymbol{u})+(\tilde{\boldsymbol{p}}, \boldsymbol{u})_{X}+C
$$

where $C$ is a constant. We first compute $\boldsymbol{v}^{k}$ using the following semi-implicit scheme derived from [9].

$$
v_{i}^{\ell+1}=\frac{v^{\ell}+\tau \nabla\left(\nabla \cdot v_{i}^{\ell}-\tilde{p}_{i}\right)}{1+(\tau / g)\left[\sum_{i=1}^{3}\left|\nabla\left(\nabla \cdot v_{i}^{\ell}-\tilde{p}_{i}\right)\right|_{2}^{2}\right]^{1 / 2}}, \quad i=1,2,3,
$$

where $\tau>0$. With $\boldsymbol{v}^{k}$ computed using (3.5) and the extremality condition we recover the minimizer of Subproblem 1

$$
\bar{u}_{i}^{k}=\frac{1}{r}\left(\nabla \cdot v_{i}^{k}-\tilde{p}_{i}\right), \quad \text { i.e. } \quad u_{i}^{k}=f_{i}-p_{i}^{k-1}+\frac{1}{r}\left(\nabla \cdot v_{i}^{k}-s_{i}^{k}\right), \quad i=1,2,3 .
$$

\subsection{Solution of subproblem 2}

The functional $\boldsymbol{p} \mapsto \mathscr{L}_{r}\left(\boldsymbol{u}^{k}, \boldsymbol{p} ; \boldsymbol{s}^{k}\right)$ can be rewritten as

$$
\Phi_{2}(\boldsymbol{p})=(\lambda+r / 2)\|\boldsymbol{p}\|_{L^{2}}^{2}+\left(\boldsymbol{s}^{k}+r\left(\boldsymbol{u}^{k}-\boldsymbol{f}\right), \boldsymbol{p}\right)_{X}+C,
$$

where $C$ is a constant. We deduce the solution of the minimization subproblem in $\boldsymbol{p}$

$$
\boldsymbol{p}^{k}=-\left(\boldsymbol{s}^{k}+r\left(\boldsymbol{u}^{k}-\boldsymbol{f}\right)\right) /(r+2 \lambda) .
$$

With the results above, we can now present the Uzawa block relaxation algorithms for the $T V_{g}+L^{2}$ model.

\subsection{Uzawa block relaxation algorithms}

\section{Algorithm TVL2/UBR2}

Initialization. $\boldsymbol{p}^{-1}, \boldsymbol{s}^{0}$ and $r>0$ given.

Iteration $k \geq 0$. Compute successively $\boldsymbol{u}^{k}, \boldsymbol{p}^{k}$ and $\boldsymbol{s}^{k}$ as follows. 
Step 1. Set $\tilde{\boldsymbol{p}}=\boldsymbol{s}^{k}+r\left(\boldsymbol{p}^{k-1}-\boldsymbol{f}\right)$ and compute $\boldsymbol{v}^{k}$ with (3.5).

Compute $\boldsymbol{u}^{k}$

$$
u_{i}^{k}=f_{i}-p_{i}^{k-1}+\frac{1}{r}\left(\nabla \cdot v_{i}^{k}-s_{i}^{k}\right), \quad i=1,2,3 .
$$

Step 2. Update the Lagrange multiplier

$$
\boldsymbol{s}^{k+1 / 2}=\boldsymbol{s}^{k}+\frac{r}{2}\left(\boldsymbol{u}^{k}+\boldsymbol{p}^{k-1}-\boldsymbol{f}\right) .
$$

Step 3. Compute $\boldsymbol{p}^{k}$

$$
\boldsymbol{p}^{k}=-\left(\boldsymbol{s}^{k}+r\left(\boldsymbol{u}^{k}-\boldsymbol{f}\right)\right) /(r+2 \lambda)
$$

Step 4. Update the Lagrange multiplier

$$
\boldsymbol{s}^{k+1}=\boldsymbol{s}^{k+1 / 2}+\frac{r}{2}\left(\boldsymbol{u}^{k}+\boldsymbol{p}^{k}-\boldsymbol{f}\right) .
$$

\section{Augmented Lagrangian methods for the $T V+L_{g}^{1}$ model}

In this part, we give some elements for the resolution of the $T V+L_{g}^{1}$ model where $L_{g}^{1}$ is a vectorial spatially adaptive data term. The function $\boldsymbol{g}(x)$ is then here chosen as a vectorial function $\boldsymbol{g}(x)=\left(g_{1}(x), g_{2}(x), g_{3}(x)\right)$. The new model $T V+L_{g}^{1}$ corresponds to the minimization of the functional (2.2). For this minimization problem, we apply the same kind of minimization procedure as in the previous section and as in [8] but, in the development of the solution of subproblem 1, we have the next change:

$$
v_{i}^{\ell+1}=\frac{v^{\ell}+\tau \nabla\left(\nabla \cdot v_{i}^{\ell}-\tilde{p}_{i}\right)}{1+(\tau / \lambda)\left[\sum_{i=1}^{3}\left|\nabla\left(\nabla \cdot v_{i}^{\ell}-\tilde{p}_{i}\right)\right|_{2}^{2}\right]^{1 / 2}}, \quad i=1,2,3,
$$

where $\tau>0$.

The algorithm then becomes:

\section{Algorithm TVL1g/UBR2}

Initialization. $\boldsymbol{p}^{-1}, \boldsymbol{s}^{0}$ and $r>0$ given.

Iteration $k \geq 0$. Compute successively $\boldsymbol{u}^{k}, \boldsymbol{p}^{k}$ and $\boldsymbol{s}^{k}$ as follows.

Step 1. Set $\tilde{\boldsymbol{p}}=\boldsymbol{s}^{k}+r\left(\boldsymbol{p}^{k-1}-\boldsymbol{f}\right)$ and compute $\boldsymbol{v}^{k}$ with (4.1).

Compute $\boldsymbol{u}^{k}$

$$
u_{i}^{k}=f_{i}-p_{i}^{k-1}+\frac{1}{r}\left(\nabla \cdot v_{i}^{k}-\boldsymbol{s}_{i}^{k}\right), \quad i=1,2,3 .
$$

Step 2. Update the Lagrange multiplier

$$
\boldsymbol{s}^{k+1 / 2}=\boldsymbol{s}^{k}+\frac{r}{2}\left(\boldsymbol{u}^{k}+\boldsymbol{p}^{k-1}-\boldsymbol{f}\right) .
$$

Step 3. Compute $\boldsymbol{p}^{k}$

$$
p_{i}^{k}= \begin{cases}0 & \text { if }\left|s_{i}^{k}+r\left(u_{i}^{k}-f_{i}\right)\right| \leq g_{i}, \\ f_{i}-u_{i}^{k}-\frac{1}{r}\left[s^{k}-g_{i} \frac{s_{i}^{k}+r\left(u_{i}^{k}-f_{i}\right)}{\left|s_{i}^{k}+r\left(u_{i}^{k}-f_{i}\right)\right|}\right] & \text { if }\left|s_{i}^{k}+r\left(u_{i}^{k}-f_{i}\right)\right| \geq g_{i} .\end{cases}
$$

Step 4. Update the Lagrange multiplier

$$
\boldsymbol{s}^{k+1}=\boldsymbol{s}^{k+1 / 2}+\frac{r}{2}\left(\boldsymbol{u}^{k}+\boldsymbol{p}^{k}-\boldsymbol{f}\right) .
$$




\section{$5 \quad$ Numerical experiments}

In the numerical experiments, for the fixed-point algorithm (3.5), the step size is $\tau=.125$ and the tolerance for the relative error is 1 . Numerical experiments not reported here for space reasons prove that the $U B R$ scheme is robust towards the choice of the penalty parameter $r$. In this paper, we provide two main examples to show the potential of spatially varying regularization and data terms.

\subsection{Spatially varying regularization term, the $T V_{g}+L^{2}$ model, for old parchments restoration}

In this subsection, we propose to test the impact of the function $g$ within the framework of parchments restoration. In order to restore the background of these images while preserving the text, we propose to define a well-adapted g function in the weighted total variation term. On the basis of several tests, we finally choose to introduce an information on the vectorial gradient through the use of a mask function. In order to do so, we first compute the image corresponding to the norm of the gradient of each channel. We then perform a basic classification of each gradient image by partitioning its histogram into $n b_{\text {classes }}$ by minimizing the sum of the class variances. The number of classes was chosen equal to 3 and we pay attention to the minimum class of each gradient image, namely $C_{\min }\left(\left|\nabla f_{i}\right|\right)$ in order to detect non significant gradients. The proposed mask function is then the following:

$$
m(x)=\left\{\begin{array}{ccc}
\alpha_{n} & \text { if } & x \in\left(C_{\min }\left(\left|\nabla f_{1}\right|\right) \cap C_{\min }\left(\left|\nabla f_{2}\right|\right) \cap C_{\min }\left(\left|\nabla f_{3}\right|\right)\right) \\
\alpha & \text { elsewhere }
\end{array}\right.
$$

We choose $\alpha_{n}=1$ and $\alpha=0.001$ in order to uppermost smooth the pixels of smaller gradients. We then take $g(x)=m_{\sigma}(x)$ where $m_{\sigma}(x)=G_{\sigma} * m(x)$ is a slight regularized version of $m(\sigma=0.05)$. As far as the removing of non significant edges is concerned in old parchments, this adjunction of a weighted TV leads to interesting results that can not be obtained using a classical TV regularization term. In Figure 5.1, we show an example of restoration of an old parchment using $g=1$ (Figure 5.1.(b)) and the function $g=m_{\sigma}(x)$ (Figure 5.1.(c)). The parameter $\lambda$ was chosen in order to visually obtain the best results $\left(\lambda=3\right.$ when $g=1$ and $\lambda=0.2$ when $\left.g=m_{\sigma}(x)\right)$. When choosing $g=1$, the parameter $\lambda$ is difficult to tune. Indeed choosing a small value for this parameter leads to a global smoothing of the image and does not allow a preservation of the characters while choosing a high value does not smooth enough the background. On the contrary, when using the weighted TV term, the impact of the parameter $\lambda$ is less important. We can then choose a small value in order to smooth the background while preserving the important information.

This first experimental result is here to illustrate the behaviour of the function $g$ in the weighted total variation term. Further experiments are needed in order to validate this term for the specific application of old parchments restoration and analysis. It could also be interesting to test some different space colors than RGB in order to improve these first results.

\subsection{Spatially varying data term, the $T V+L_{g}^{1}$ model, for impulse noise removal}

In order to test the potential of such a vectorial varying data term, we first propose to test it on simulated noisy images where an impulse noise is added on each color channel. To achieve this, we introduce the vector-valued function $\boldsymbol{g}(x)=\left(g_{1}(x), g_{2}(x), g_{3}(x)\right)$ defined by

$$
g_{i}(x)= \begin{cases}0 & \text { if } x \in C_{i}(f) \\ 1 & \text { if not }\end{cases}
$$




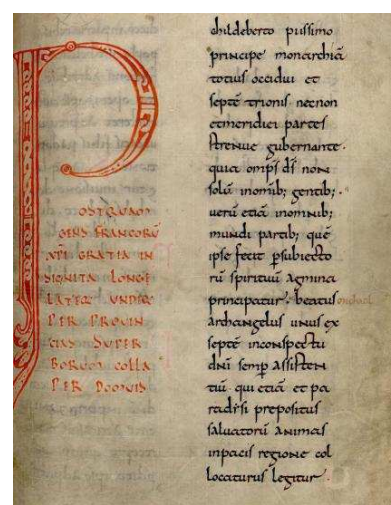

(a) Original image

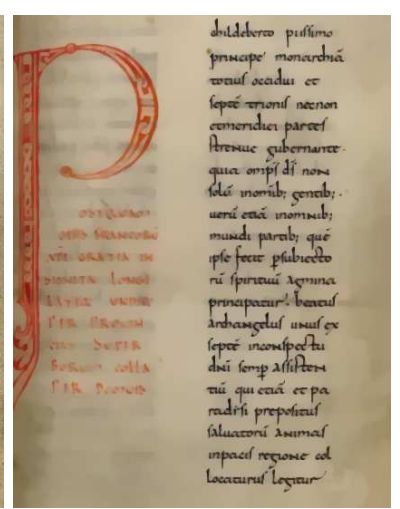

(b) Using $g=1$

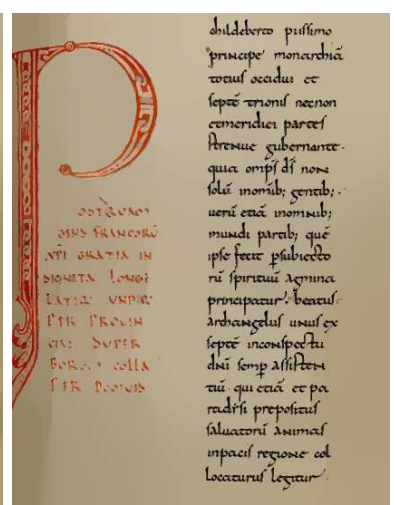

(c) Using an appropriate $g$

Fig. 1. Restoration of a parchment (a) using the weighted total variation term with $g=1$ (b) and the function $g(x)=m_{\sigma}(x)(\mathrm{c})$.

where $C_{i}(f)$ designates the set of corrupted pixels in the channel $i$. In the experiments, we consider that the corrupted pixels correspond to the minimum or maximum values of the intensity but, in the real case, an adapted noise detector should be used (see [28] for an interesting bibliography on such detectors). Figure 2 shows interestingly that the new model outperforms the $T V_{g}+L^{1}$ model where $g$ is the function proposed in [8] for salt and pepper noise removal. The spatially varying data term gives very interesting results even for high levels of noise that are only handled by very few methods [28].

\section{Conclusion}

This paper deals with color image restoration using a vectorial adaptation of the seminal ROF model. An augmented Lagrangian functional is introduced leading to Uzawa block relaxation algorithms for the minimization of both the $T V_{g}+L^{2}$ and the $T V+L_{g}^{1}$ functionals. Such algorithms are fast and easy to implement allowing a simple resolution for the minimization of such functionals. They are also robust towards the choice of the penalty parameter $r$. Once the mathematical framework was settled, we propose to test the behaviour of spatially varying regularization and data terms in the framework of color image restoration. We first propose to test the impact of the weighted total variation term through the definition of a new function $g$ that proves to be valuable for the restoration of old parchments. We use it in order to restore the background of medieval parchments while preserving the text. We obtain very good results that need to be completed by further investigations and tests. One problem that needs to be further studied is the impact of the additional term that appears when computing the PDE of such a problem. This problem was mentioned in [28]. This additional term may introduce some oscillating boundaries if the function $g$ is not smooth enough and must then be further studied and quantified. A post-processing (smoothing in the direction of the tangent of the gradient) can also be used to solve this artifact. Secondly, we also investigate the use of a spatially data varying term where the parameter $\lambda$ becomes a vectorial local function. This term can also be solved using our mathematical framework with only a slight change and leads to very good results for salt an pepper denoising. We also compare it to an adapted weighted total variation term. In this case, the spatially varying data term gives better results both visually and in terms of 
PSNR. These first tests need to be further developed for example regarding with the sensitivity to the parameters and color space. They also demonstrate that the selection of the point-wise weight function is very important and may lead to a real improvement compare to the results obtained using a simple $\mathrm{TV}+\mathrm{L}^{\tau}$ model. Our on going research is then directed towards a deeper investigation of such terms notably within the framework of old parchments restoration.

(a)
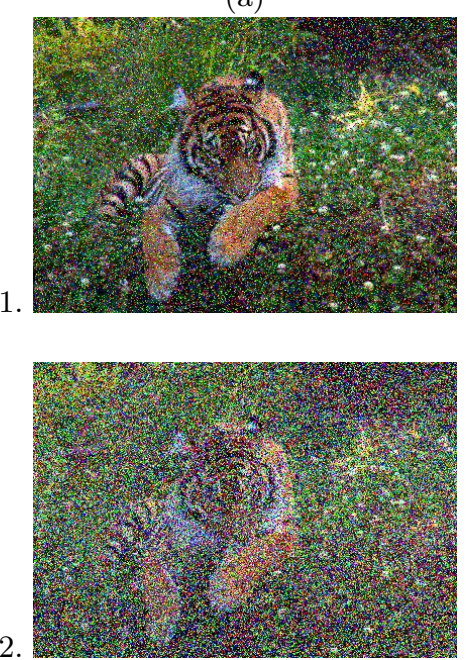

2.

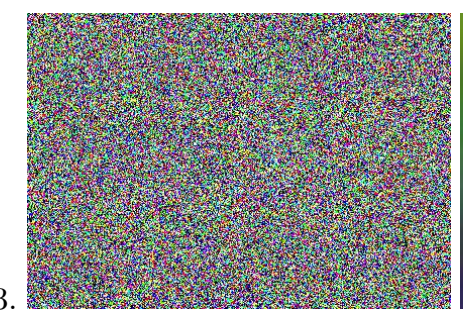

(b)

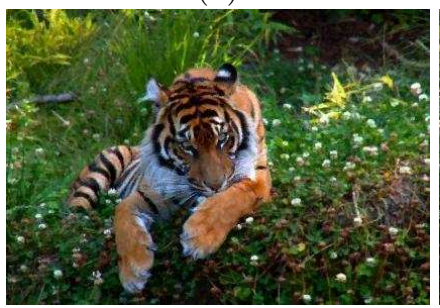

$33.5 d B, \quad 96 i t ., \quad \lambda=0.9$

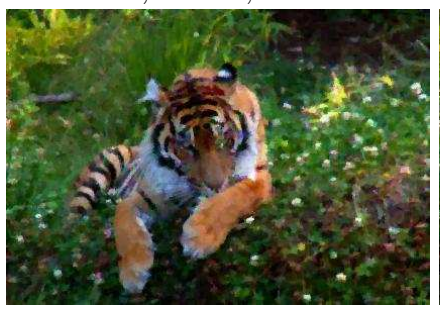

$27.6 d B, \quad 114 i t ., \quad \lambda=0.7$

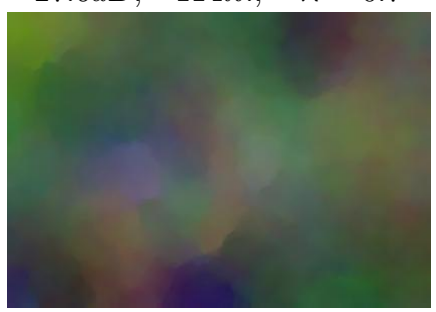

19.5dB, $\quad$ 759it.,$\quad \lambda=0.5$ (c)

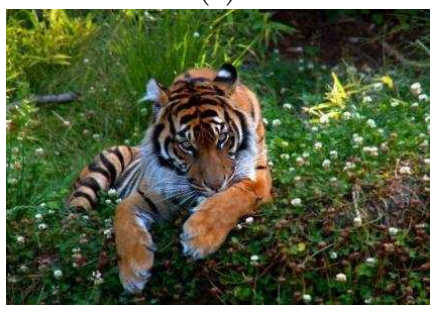

$40.4 d B, \quad 46 i t ., \quad \lambda=0.2$

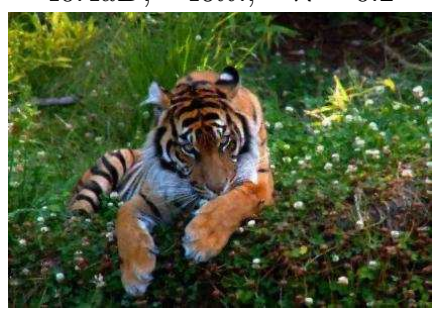

$33.9 d B, \quad 65 i t ., \quad \lambda=0.2$

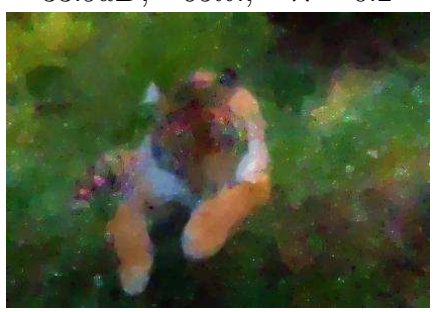

$23.0 d B, \quad 245 i t ., \quad \lambda=0.2$

Fig. 2. (a) : noisy image $(20 \%, 70 \%, 95 \%)$; (b) : restored image using $T V_{g}+L^{1}$; (c) : restored image using $T V+L_{g}^{1}$ (with the associated PSNR, number of iterations and $\lambda$ )

\section{Acknowledgements}

We would like to thank the Scriptorial Museum of Avranches (France) in link with the MRSH (Home of the Research of Human Sciences) within the University of Caen for providing us with the Medieval manuscripts of Mont St Michel. We thanks P.Y. Buard from the MRSH to make a bridge between the different scientific domains. We also thank R. Clouard and D. Tschumperlé for their useful tools for image processing (namely pandore and gmic).

\section{References}

1. Rudin, L., Osher, S., Fatemi, E.: Nonlinear total variation based noise removal algorithms. Physica D. 60 (1992) 259-268 
2. Chan, T., Golub, G., P.Mulet: A nonlinear primal-dual method for total variation-based image restoration. SIAM Journal of Scientific Computing 20 (1999) 1964-1977

3. Nikolova, M.: A variational approach to remove outliers and impulse noise. Journal of Mathematical Imaging and Vision 20 (2004) 99-120

4. Aujol, J.F., Gilboa, G., Chan, T.F., Osher, S.: Structure-texture image decomposition - modeling, algorithms, and parameter selection. International Journal of Computer Vision 67 (2006) 111-136

5. Bresson, X., Esedoglu, S., Vandergheynst, P., Thiran, J.P., Osher, S.: Fast global minimization of the active contour/snake model. Journal of Mathematical Imaging and Vision 28 (2007) 151-167

6. Chambolle, A.: Total variation minimization and a class of binary MRF models. In: Workshop on Energy Minimization Methods in Computer Vision and Pattern Recognition, LNCS. (2005) 136-152

7. Darbon, J., Sigelle, M.: Image restoration with discrete constrained total variation part I: Fast and exact optimization. Journal of Mathematical Imaging and Vision 26 (2006) 261-271

8. Koko, J., Jehan-Besson, S.: An Augmented Lagrangian Method for $\mathrm{TV} g+\mathrm{L}^{1}$-norm Minimization. Journal of Mathematical Imaging and Vision 38 (2010) Pages 182-196

9. Chambolle, A.: An algorithm for total variation minimization and applications. Journal of Mathematical Imaging and Vision 20 (2004) 89-97

10. Aujol, J.F., Chambolle, A.: Dual norms and image decomposition models. International Journal of Computer Vision 63 (2005) 85-104

11. Goldstein, T., Osher, S.: The split bregman method for $\mathrm{L}^{1}$ regularized problems. SIAM J. on Imaging Sciences 2 (2009) 323-343

12. Pock, T., Cremers, D., Bischof, H., Chambolle, A.: Global solutions of variational models with convex regularization. SIAM J. Imaging Sciences 3 (2010) 1122-1145

13. Fortin, M., Glowinski, R.: Augmented Lagrangian Methods: Application to the Numerical Solution of Boundary-Value Problems. North-Holland, Amsterdam (1983)

14. Glowinski, R., Tallec, P.L.: Augmented Lagrangian and Operator-splitting Methods in Nonlinear Mechanics. SIAM, Philadelphia (1989)

15. Blomgren, P., Chan, T.F.: Color TV: total variation methods for restoration of vector-valued images. IEEE transactions on image processing 7 (1998) 304-309

16. Sapiro, G.: Vector-valued active contours. In: International Conference on Computer Vision and Pattern Recognition. (1996) 680-685

17. Tschumperlé, D., Deriche, R.: Diffusion PDE's on vector-valued images : Local approach and geometric view-point. IEEE Signal Processing Magazine 19 (2002) 16-25

18. Di Zenzo, S.: A note on the gradient of a multi-image. In: Computer Vision, Graphics, and Image Processing. Volume 33. (1986) 116-125

19. Tschumperlé, D.: Anisotropic Diffusion PDEs. In: Digital Color Imaging. WILEY (2012) 75-121

20. Bresson, X., Chan, T.F.: Active contours based on Chambolle's mean curvature motion. In: ICIP (1). (2007) 33-36

21. Bresson, X., Chan, T.: Fast dual minimization of the vectorial total variation norm and applications to image processing. Inverse Problems and Imaging 2 (2008) 455-484

22. Duval, V., Aujol, J., Vese, L.: Projected gradient based color image decomposition. In: Scale Space and Variational Methods in Computer Vision (SSVM) in Lecture Notes of Computer Sciences 5567. (2009) 295-306

23. Duval, V., Aujol, J., Vese, L.: Mathematical modeling of textures: Application to color image decomposition with a projected gradient algorithm. Journal of Mathematical Imaging and Vision 37 (2010) 232-248

24. Dong, Y., Hintermüller, M., Montserrat Rincon-Camacho, M.: A multi-scale vectorial L ${ }^{\tau}$-TV framework for color image restoration. International Journal of Computer Vision 92 (2011) 296-307

25. Goldlücke, B., Cremers, D.: An approach to vectorial total variation based on geometric measure theory. In: Int. Conf. on Computer Vision and Pattern Recognition. Volume 106. (2010) 327-333

26. Grasmair, M.: Locally adaptive total variation regularization. In: Scale Space and Variational methods in computer Vision. (2009) 331-342

27. Jehan-Besson, S., Koko, J.: Fast dual minimization of weighted TV + L1 norm for salt and pepper noise removal. In: VISAPP, Angers, France (2010)

28. Duval, V.: Variational and non-local methods in image processing: a geometric study. PhD thesis, Ecole Nationale Supérieure des Télécommunications, Telecom Paris Tech (2011) 